\title{
ANTIFUNGAL ACTIVITY OF NANOSTRUCTURED FILMS WITH SILVER NANOPARTICLES
}

\author{
Rocha, J. S. ${ }^{1 *}$; Menezes, E. A. ${ }^{3}$; Cunha, F. A. ${ }^{2},{ }^{3}$; Fechine, P. B. A. ${ }^{2}$ \\ ${ }^{1}$ Department of Materials Science and Engineering, Universidade Federal do Ceará, Fortaleza, Ceará, Brazil \\ ${ }^{2}$ Department of Analytical Chemistry and Physical Chemistry, Universidade Federal do Ceará, Fortaleza, Ceará, Brazil \\ ${ }^{3}$ Department of Clinical and Toxicological Analysis, Universidade Federal do Ceará, Fortaleza, Ceará, Brazil \\ *janaina.s@fisica.ufc.br
}

The objective of this work was to produce, characterize and evaluate antifungal activity of agarose nanostructured films with silver nanoparticles using glucose as a reducing agent and sodium dodecyl sulfate (SDS) as stabilizer. The AgNPs were synthesized and purified using ultracentrifugation. The characterization was performed using UV-visible spectroscopy in the range of 300-700 nm, Spectroscopy Fourier Transform Infrared (FT-IR), X-ray diffraction, determination of particle size and scanning electron microscopy. The film was produced with built-agarose and AgNPs were incorporated by ex-situ technique. The XRD patterns show that the AgNP are also crystalline in nature. The peaks were assigned to diffraction from the (111), (200), (220), (311) and (222) planes of face-centered cubic (fcc) silver, which were in good agreement with reference to the unit cell of the fcc structure. The film showed very good looking uniform with relief, good transparency and resistance. To evaluate the antifungal activity of films, 20 strains of Candida albicans were tested by the disk diffusion method. There was formation of a zone of inhibition, ranging in average $19.1 \mathrm{~mm}$. Preliminary results of nanostructured films with AgNPs proved an excellent antifungal agent. 